\title{
émulations
}

Laurent Di Filippo, Hélène François, Anthony Michel (dir.) - La position du doctorant. Trajectoires, engagements, réflexivité

\section{Pauline Neveu}

Émulations - Revue de sciences sociales

2016, « Comptes rendus critiques, En ligne »

Article disponible à l'adresse suivante

https://ojs.uclouvain.be/index.php/emulations/article/view/ 7013

\section{Pour citer cet article}

Nicolas Riffault, « Laurent Di Filippo, Hélène François, Anthony Michel (dir.) — La position du doctorant. Trajectoires, engagements, réflexivité », Émulations, en ligne. Mise en ligne le 22 mars 2016.

DOI : 10.14428/emulations.cr.005

Distribution électronique : Université catholique de Louvain (Belgique) : ojs.uclouvain.be

(c) Cet article est mis à disposition selon les termes de la Licence Creative Commons Attribution, Pas d'Utilisation Commerciale 4.0 International. http://creativecommons.org/licenses/by-nc/4.0/

Éditeur : Émulations - Revue de sciences sociales / Presses universitaires de Louvain https://ojs.uclouvain.be/index.php/emulations

ISSN électronique : $1784-5734$

UCL PRESSES

UNIVERSITAIRES

DE LOUVAIN 


\section{Laurent Di Filippo, Hélène François, An- thony Michel (dir.) - La position du doc- torant. Trajectoires, engagements, ré- flexivité}

Pauline Neveu ${ }^{1}$

Recensé : Laurent Di Filippo, Hélène François, Anthony Michel (dir.), La position du doctorant. Trajectoires, engagements, réflexivité, Nancy, Presses universitaires de Nancy, 2012, 210 p.

Alors que le nombre d'étudiants débutant un doctorat augmente d'année en année en France ${ }^{2}$, la littérature scientifique peine à produire autre chose que des traités méthodologiques prodiguant des conseils, principalement, à propos de la rédaction ou de la publication. L'ouvrage collectif La position du doctorant. Trajectoire, engagements, réflexivité présente et interroge - à travers l'angle de la réflexivité scientifique - des processus de positionnement et d'apprentissage rarement abordés, et qui sont pourtant inhérents à la condition doctorale. La réflexivité est comprise comme un retour sur soi, et non une contemplation narcissique. Selon les auteurs, «Être capable de définir le point de vue de l'observation et ses limites permet une meilleure interprétation des phénomènes qui occupent le chercheur et une meilleure appréhension des dites productions par leurs destinataires » (p.9). Le défi des écrits collectifs réside dans l'édification d'un rendu cohérent et harmonieux. Les neuf contributions qui structurent l'ouvrage réussissent à dresser un panorama des divers emplois de la réflexivité à cette étape charnière qu'est le doctorat. Pour ce faire, les auteurs, en se prenant eux-mêmes pour objet d'étude, se questionnent sur les innombrables prises de position qu'ils ont dû opérer en tant que jeunes chercheurs, qu'elles soient disciplinaires, heuristiques, méthodologiques, ou encore, éthiques. Ainsi, le recueil s'articule autour de quatre problématiques complémentaires sur lesquelles pivote la réflexion des contributeurs : se situer en tant que chercheur dans les champs scientifiques, déconstruire son sujet, interroger son expérience personnelle, et enfin, faire le difficile lien entre pratique et

\footnotetext{
${ }^{1}$ Doctorante en sociologie à l'INRS - Urbanisation, Culture et Société, Université du Québec à Montréal.

${ }^{2}$ Les chiffres de l'INSEE indiquent qu'il y aurait, en France, 7,2 milliers d'inscrits au doctorat 1990 pour 11.8 en 2012: http://www.insee.fr/fr/mobile/etudes/document.asp?reg_id=0\&ref_id=T15F102.
} 
théorie. Faisant la part belle à la transdisciplinarité, des sociologues, historiens, linguistes, mais aussi une japonologue, ou encore une chercheuse en arts plastiques, tentent de rendre compte de leur parcours réflexif doctoral.

Se situer semble s'imposer comme l'étape initiale à toute réflexivité. Les trois premières contributions invitent donc le lecteur à s'interroger sur les legs scientifiques qu'il a reçus, ainsi que les réinterprétations qu'il est possible d'en faire. Il s'agit de se positionner en tant que chercheur dans l'espace des savoirs. Ces différents ajustements conceptuels, éthiques, et disciplinaires, sont des choix nécessaires, bien que difficiles, afin de se constituer une identite de chercheur. Les contributions de Fanny Martin et d'Audrey Bonjour s'attachent à présenter les défis inhérents à certains terrains de recherche, respectivement, le monde du basket et du handicap. La première suggère à quel point un mouvement réflexif peut devenir un chemin pour mieux aborder le terrain, particulièrement dans la présentation de soi aux enquêtés. Tandis que la seconde présente les prises de position qu'elle a été amenée à faire en tant que doctorante étudiant le handicap. Nous retiendrons surtout ses choix éthiques sur son sujet qualifié de «sensible », ce qui amène le lecteur à s'interroger sur le niveau d'affects et de polémique que peuvent susciter certains objets de recherche. La contribution d'Hélène François démontre, quant à elle, l'importance de déconstruire l'héritage institutionnel d'une discipline. Cette dernière s'est retrouvée en décalage avec les études civilisationnistes qui, par coutume, adoptent un angle d'approche littéraire, et non historique comme souhaitait le faire la doctorante dans sa démarche. Faire la généalogie de sa discipline lui a permis de la décloisonner, de diversifier ses méthodes, et ainsi, de trouver sa manière de faire la recherche. Dans tous les cas présentés, on saisit l'importance de remettre en question des enseignements scientifiques, parfois considérés comme acquis, afin de se constituer en tant que chercheur. Si saisir sa localisation sur la carte universitaire est important, la démarche réflexive réclame d'autres phases : notamment, une déconstruction du sujet de recherche. En effet, repenser, réinterroger l'objet d'analyse scientifique qu'on a créé, constitue une façon de dévoiler certaines dimensions, qui, bien qu'extérieures à la recherche, éclairent son déroulement. Comme l'expose Anthony Michel, un sujet, même s'il est choisi pour des raisons scientifiques, est aussi partiellement sélectionné : des cours, une rencontre avec un directeur de thèse, ou plus simplement une curiosité intellectuelle attachent le chercheur à un thème spécifique plutôt qu'à un autre. Tout comme annoncé dans l'introduction de l'ouvrage, ce lien doit être explicité afin de fournir les conditions de production du savoir. De plus, comme le rappelle la japonologue Magali Bugne, il s'agit d'un bon moyen pour maintenir une vigilance épistémologique nécessaire. En effet, les héritages scientifiques et théoriques des orientalistes - ces penseurs ayant une forte tendance colonialiste - ainsi que sa formation en langues, on fait basculer la doctorante dans une forme d'idéalisation de la culture japonaise. Grâce à un effort réflexif, la chercheuse a retravaillé et déconstruit scientifiquement cette glorification de son objet. Ainsi, le retour sur le sujet, et de fait, sur les chemins qui y ont mené est central dans un processus réflexif : cela met en lumière des 
conceptions et des perceptions difficilement accessibles sans auto-analyse, et pourtant constitutives du processus intellectuel de la recherche.

Le troisième mouvement réflexif — qui est sûrement le plus ardu pour les doctorants occidentaux qui ont hérité des valeurs positivistes du XIX ${ }^{\mathrm{e}}$ siècle dans leurs disciplines - consiste en la reconnaissance heuristique du savoir personnel des chercheurs. Prendre cette caractéristique en compte revient à reconnaître la pertinence de l'expérience individuelle, psychologique et émotionnelle de l'individu-chercheur comme source de données, mais aussi, comme une méthode d'analyse et de compréhension. Les auteurs démontrent avec justesse que l'expérience individuelle du chercheur, si elle n'est pas clairement traitée, peut se révéler être un frein et un biais considérable à la compréhension intellectuelle du monde. Tandis que si elle est bien intégrée au processus de pensée, elle devient un gage de scientificité. Sahand Aleboyeh, doctorant étudiant le handicap et étant lui-même porteur d'une déficience, donne tout son sens à cet emploi de la réflexivité. Durant son travail de mémoire, il a adopté une posture visant à la neutralité absolue. Il explique : « je craignais que l'on me voie comme incapable de travailler sur cette thématique, car j'étais trop proche d'elle » (p. 127). Il réalisera au début de son doctorat que sa situation lui permet des accès et une compréhension que d'autres chercheurs n'auraient pas. Ainsi, il était plus à même de gérer ses rapports aux acteurs associatifs et administratifs, ou sa réaction devant un corps handicapé. De la même façon, Albinou Ndecky, chercheur polyglotte qui étudie sa langue maternelle, démontre l'évolution de sa posture scientifique en parallèle avec son parcours de vie, l'association des deux l'ayant finalement guidée vers la linguistique et le choix d'un terrain de recherche à quelques kilomètres de son village natal. Cet exercice de dévoilement de son expérience personnelle apparaît délicat puisqu'il réclame une grande capacité de clarté avec soi-même. Or, rien dans la formation en recherche (si l'on exclut celle des psychologues et psychiatres) ne prépare à cela. Néanmoins, ce défi témoigne des caractéristiques personnelles que peut revêtir la découverte scientifique, caractéristiques que tout doctorant se doit de traiter durant son parcours.

Le dernier aspect traité par les auteurs concerne une tension structurante dans la recherche, à savoir, la collaboration heuristique entre la théorie et la pratique. À cette occasion, Aurélie Michel décrit la position si particulière du chercheur en arts plastiques. Ce dernier se doit de manipuler des théories concernant la production artistique, mais aussi d'engendrer des œuvres concrètes. Ce rapprochement des deux dimensions relève d'un jonglage constant entre références théoriques et acte de création lui-même. L'auteure parle des tendances schizophréniques du chercheur-plasticien qui se doit de revenir fréquemment sur son acte créatif afin de comprendre et de formuler une théorie de sa production artistique. Une volonté d'articulation entre le concret du terrain, l'individualité de la réflexivité, et l'abstraction de la théorisation, s'est aussi présentée à Laurent Di Filippo lors de son étude sur le jeu vidéo. Ce dernier soutient qu'être un joueur lui offre des renseignements et des interprétations atypiques et inac- 
cessibles à d'autres chercheurs. Ces informations colorent son analyse d'une dimension concrète et enrichissent sa théorisation. Ainsi, des données internes, parfois affectives, sont présentées comme pouvant faire travailler ensemble théorie et pratique. Par exemple, le sociologue parvient à traiter scientifiquement l'émotion ressentie lorsqu'il est émerveillé par la musique d'un des territoires du MMORPG ${ }^{3}$ qu'il analyse. Comme il l'explique : «Ce genre d'occasions doit alerter le chercheur sur l'effet recherché par les concepteurs et comment ces derniers cherchent à produire un sens particulier à travers les multiples éléments présents en jeu » (p. 182). Systématiser le lien entre théorie et pratique via la réflexivité est ardu, car, en plus du grand écart intellectuel que cela sous-entend, cette relation n'est transmissible bien souvent qu'à travers des exemples. Les deux articles réussissent à présenter leurs cas de façon claire et convaincante, et amènent le lecteur à s'inspirer de ces démarches pour sa propre recherche. Car l'originalité de cet ouvrage, outre son approche transdisciplinaire, se loge dans une forte exigence pédagogique. Les articles fournissent de judicieux exemples où la réflexivité s'est vue opérationnalisée scientifiquement. De cette façon, le lecteur peut s'inspirer et apprendre des expériences évoquées. À noter que le livre se conclut par une liste de références bibliographiques sélectionnées et commentées par les auteurs. On apprécie cette volonté de poursuivre la réflexion de façon plus précise et informée qu'avec une bibliographie classique qui laisse les titres parler d'eux-mêmes.

Finalement, que retenir de contributions si individuelles et si diverses? Très certainement que la réflexivité n'est pas un luxe heuristique et qu'il est fructueux, voire, primordial de mobiliser un retour sur soi lors de sa recherche. Or, cette prise de conscience ne peut se faire que dans une forme d'auto-analyse personnelle, disciplinaire, méthodologique ou encore éthique, où le scientifique tentera de se replacer, en tant qu'observateur dans l'observation, pour faire des choix scientifiquement pertinents. 3 «Massively multiplayer online role-playing game», en français : «Jeu de rôle en ligne mas-
sivement multijoueur ». 